\title{
GENETIC SUPPORT OF CARBAPENEMASES IN DOUBLE CARBAPENEMASE PRODUCER KLEBSIELLA PNEUMONIAE ISOLATED IN THE ARABIAN PENINSULA
}

\author{
Amna E. Al-Baloushi ${ }^{1,2}$, Tibor PÁl ${ }^{1}$, AKela GHAZAWI ${ }^{1}$ and \\ AGNES SONNEVEND ${ }^{1 *}$ \\ ${ }^{1}$ Department of Microbiology and Immunology, College of Medicine and Health \\ Sciences, United Arab Emirates University, Al Ain, United Arab Emirates \\ ${ }^{2}$ Microbiology Laboratory, Al Ain Hospital, Al Ain, United Arab Emirates
}

(Received: 20 September 2017; accepted: 27 November 2017)

Enterobacteriaceae co-producing NDM- and OXA-48-type carbapenemases were encountered in higher frequency in the United Arab Emirates (UAE) than in the neighboring countries in our earlier study. The aim of this investigation was to characterize the seven double carbapenemase producer Klebsiella pneumoniae found in the region to assess factors contributing to their emergence. Three K. pneumoniae ST14 isolated in the UAE harboring $b l a_{\mathrm{NDM}-1}$ on IncHIlb and $b l a_{\mathrm{OXA}-232}$ on IncColE plasmids were clonally related. Furthermore, two $K$. pneumoniae from the UAE, $\mathrm{ABC} 106$ and $\mathrm{ABC} 137$ belonged to ST307 and ST1318, respectively. ABC106 carried bla $_{\mathrm{NDM}-1}$ on IncHI1b, and bla $a_{\mathrm{OXA}-162}$ on IncL/M plasmids, whereas $\mathrm{ABC} 137$ possessed $b l a_{\mathrm{NDM}-1}$ on IncX3 and $b l a_{\mathrm{OXA}-48}$ on IncL/M plasmids. The double carbapenemase-producing K. pneumoniae from Oman (OMABC109) and Saudi Arabia (SA54) belonged to ST11 and ST152, respectively. OMABC109 harbored $b l a_{\mathrm{NDM}-1}$ on an IncHI1b plasmid highly similar to the NDM-plasmid of ABC106 and carried a chromosomally coded bla $a_{\text {OXA-181 }}$ located on Tn2013. SA54 possessed a $b l a_{\mathrm{NDM}-1}$ on an IncFIb/FII plasmid and a $b l a_{\mathrm{OXA}-48}$ on an IncL/M plasmid. Based on these findings, clonal spread and horizontal transfer of carbapenemase genes located on transposons or self-transmissible plasmids contributed equally to the emergence of double carbapenemase-producing Enterobacteriaceae in the region.

Keywords: Enterobacteriaceae, carbapenem resistance, NDM- and OXA-48type carbapenemases, Middle East

*Corresponding author; E-mail: agnes_sonnevend@uaeu.ac.ae 


\section{Introduction}

The emergence and rapid spread of carbapenem-resistant Enterobacteriaceae (CRE) is a major global concern [1]. In the majority of cases carbepenem resistance is due to the production of carbapenemases with varying levels of hydrolyzing activity against other beta-lactams. Carbapenemase enzymes belong either to Ambler class A, B, or D beta-lactamases. Their geographical distribution varies, but in general the most common ones are the KPC-, IMP-, VIM-, NDM-, and OXA-48-type enzymes. These enzymes often coded by genes located, as part of composite transposons, on conjugative plasmids that further facilitate their horizontal transfer [2].

It is likely that this mobile nature of carbapenemase genes has contributed to the emergence of Enterobacteriaceae, mostly Klebsiella pneumoniae, producing two or occasionally even three different carbapenemase genes. In Greece, where such isolates were first encountered, common combination of carbapenemases includes strains co-producing KPC-2 and various alleles of VIM [3, 4]. In other parts of the world, including the United States, Denmark, Turkey, Singapore, and India, strains expressing NDM-type carbapenemases together with OXA-48-like enzymes had emerged [5-9].

In a previous study investigating the molecular epidemiology of CRE in the Arabian Peninsula, we encountered five Klebsiella pneumoniae isolates co-producing NDM- and OXA-48-type carbapenemases in the United Arab Emirates (UAE) representing $8.9 \%$ of CRE isolates of this country. This was a considerable higher proportion than in other countries of the region [i.e., $1.9 \%$, $0 \%$, and $1.6 \%$ in the Kingdom of Saudi Arabia (KSA), Oman, and Kuwait, respectively] [10]. The aim of this study was to subject these five Emirati strains and the two isolates from the KSA and from Oman producing double carbapenemase to detailed molecular analysis to reveal whether the higher rate of such isolates in the UAE is due to a clonal expansion or, alternatively, to the emergence of unrelated strains.

\section{Methods}

\section{Bacterial strains}

The strains were encountered between April 2009 and 2013 during a previously published study [10]. The most important features of the isolates are summarized in Table I. 


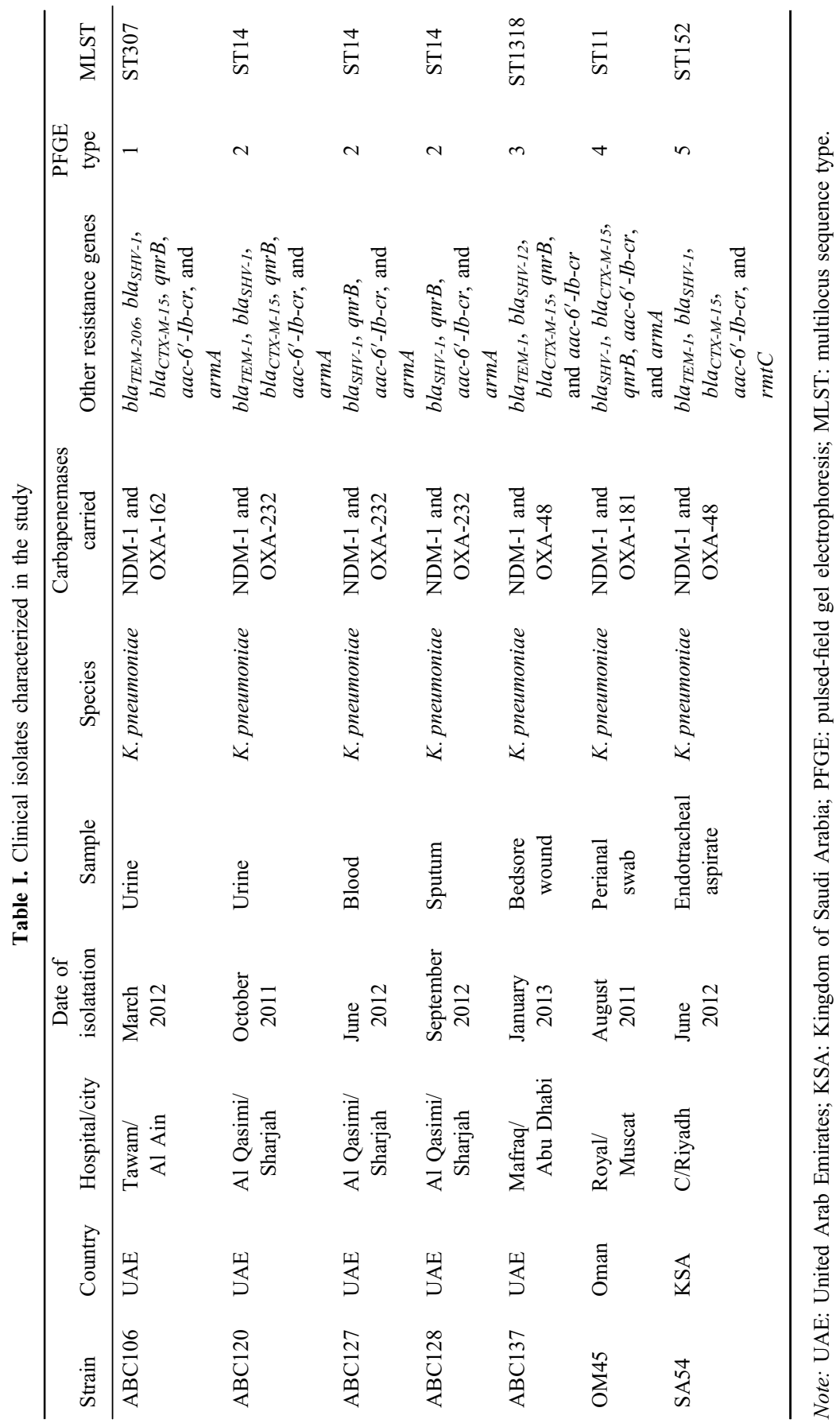


Antibiotic susceptibility assays

Susceptibility to cefotaxime, ceftazidime, aztreonam, ertapenem, meropenem, imipenem, ciprofloxacin, gentamicin, amikacin, trimethoprime/ sulfamethoxazole, tetracycline, chloramphenicol, and colistin was tested by broth microdilution, whereas tigecycline resistance was assessed by agar dilution [11]. For interpretation, the CLSI clinical breakpoints were used [11], with the exception of colistin and tigecycline interpreted by the EUCAST criteria (www.eucast.org).

\section{Molecular characterization of the strains}

The multilocus sequence type (MLST) of the isolates and the macrorestriction pattern of the XbaI-digested genomic DNA were established as described earlier $[12,13]$.

Resistance genes $\left(\right.$ bla $_{\mathrm{TEM}}$, bla $_{\mathrm{CTX}-\mathrm{M}}$, bla $_{\mathrm{SHV}}, b l a_{\mathrm{PER}}, b l a_{\mathrm{AmpC}}, b l a_{\mathrm{NDM}}$, $b l a_{\mathrm{OXA}-48-1 i k e}, b l a_{\mathrm{KPC}}, b l a_{\mathrm{VIM}}, b l a_{\mathrm{IMP}}, a r m A, r m t A, r m t B, r m t C, r m t D, q n r S, q e p A$, and aac6-1b-cr) were detected by PCR, as previously described [10, 12, 13]. The specific alleles of $b l a_{\mathrm{TEM}}, b l a_{\mathrm{SHV}}$, and $b l a_{\mathrm{CTX}-\mathrm{M}}$ beta-lactamase genes were determined by direct sequencing of the respective amplicons.

\section{Characterizations of carbapenemase-bearing plasmids}

Plasmids were routinely detected and sized by the alkaline lysis method of Kado and Liu [14] using the episomes in E. coli V517 [15] and E. coli 39R861 [16] as molecular mass standards. A Na-azide-resistant derivative of E. coli J53 $\left(\mathrm{J} 53_{\mathrm{RAZ}}\right)$ was used as recipient in conjugation. Competent cells of $E$. coli $\mathrm{J} 53_{\mathrm{RAZ}}$ or of $E$. coli $\mathrm{DH} 5 \alpha$ were used in transformation experiments. bla $a_{\mathrm{NDM}}$ plasmidscarrying derivatives were selected on plates containing $8 \mathrm{mg} / \mathrm{L}$ ceftazidime and $150 \mathrm{mg} / \mathrm{L} \mathrm{Na-azide,} \mathrm{whereas} \mathrm{those} \mathrm{carrying}$ bla $_{\text {OXA-48-like-bearing plasmids were }}$ selected on plates supplemented with $0.5 \mathrm{mg} / \mathrm{L}$ ertapenem, $200 \mathrm{mg} / \mathrm{L}$ dipicolinic acid, and $150 \mathrm{mg} / \mathrm{L}$ Na-azide. Transfer experiments were considered successful, if a single plasmid containing derivative was obtained. This was confirmed by PCR targeting the respective carbapenemase gene and by Southern blotting and hybridization of the plasmid electrophoresis gel. Attempts to detect extra-large plasmids were carried out by S1 nuclease digestions as described [13]. PCR-based replicon typing was done as described earlier [17, 18]. Southern blotting and hybridization of plasmids separated by regular gel electrophoresis or after $\mathrm{S} 1$ digestion was carried out as previously described [12]. 
Plasmid restriction fragment length polymorphism (RFLP), applying $B a m H I, S m a \mathrm{I}$, and $X b a \mathrm{I}$ restriction endonucleases, was used to compare plasmids of the same molecular mass and incompatibility type, carrying the same carbapenemase with identical flanking regions in clonally unrelated strains [12].

Characterization of the genetic environment of bla $a_{N D M}$ and bla ${ }_{O X A-48-l i k e}$ genes

The genetic scaffold of $b l a_{\mathrm{NDM}}$ and $b l a_{\mathrm{OXA}}$ was determined by PCR mapping, using crude plasmid extracts as a DNA template from the clinical isolates as described earlier [12]. This approach failed to map the $b l a_{\mathrm{NDM}}$ surrounding of $K$. pneumoniae $\mathrm{OMABC} 109$ and $\mathrm{ABC} 120$, therefore, pOMABC109-NDM and $\mathrm{pABC} 120-\mathrm{NDM}$ were digested with HindIII restriction endonuclease, and the fragments were cloned into pUC19 and transformed into E. coli DH5 $\alpha$. The bla $\mathrm{NDM}^{-}$ bearing fragments cloned into pUC19 were selected on Triptic Soy Agar medium containing $8 \mathrm{mg} / \mathrm{L}$ ceftazidime. The $b l a_{\mathrm{NDM}^{-}}$-containing pUC19 plasmids were purified with Plasmid Mini Kit (Qiagen NV, Venlo, The Netherlands), and used as templates for sequencing the structures surrounding the $b l a_{\mathrm{NDM}}[19]$.

\section{Sequencing}

PCR amplicons were purified with Wizard $^{\circledR}$ SV Gel and PCR Clean-Up System (Pomega, USA). Plasmids were purified from single plasmid containing transconjugants or transformants using Plasmid Maxi Kit (Qiagen). Sequencing was performed with the Big Dye Cycle Terminator V.3.1 (Thermo Fisher Scientific, Waltham, MA, USA) on the 3130X Genetic Analyzer (Applied Biosystems) according to the manufacturer's instructions. The sequences obtained were analyzed using MEGA 4 and assembled with Clone Manager v9 software.

The assembled sequences were annotated using Sequin (http://www.ncbi. nlm.nih.gov/Sequin) and submitted to GenBank.

\section{Results}

Antibiotic susceptibility

All clinical isolates tested were multidrug resistant, i.e., resistant to all beta-lactam antibiotics tested with the exception of $\mathrm{ABC} 127$ and $\mathrm{ABC} 128$ remaining susceptible to aztreonam. The strains were also resistant to the majority of non-beta-lactam antibiotics tested. Nonetheless, all seven strains remained 
susceptible to colistin, and none of them exhibited tigecycline resistance, although tigecycline $\mathrm{MIC}$ of $\mathrm{ABC} 127, \mathrm{ABC} 128$, and $\mathrm{ABC} 137$ exceeded the clinical breakpoint of $1 \mathrm{mg} / \mathrm{L}$ (Table II).

\section{Molecular characteristics}

The seven strains exhibited five distinct pulsed-field gel electrophoresis (PFGE) patterns, with one group clustering three of the strains also sharing the ST14 sequence type (Figure 1, Table I). The remaining four isolates were not related to this group and did not show similarity by PFGE or MLST (Table I). The specific MLST types and the antibiotic-resistance genes detected in each isolate are shown in Table I.

\section{Localization of the carbapenemase genes}

To reveal their possible plasmid localization, attempts were made to transfer the $b l a_{\mathrm{NDM}}$ and $b l a_{\mathrm{OXA}-48-\text { like }}$ genes by conjugation into a Na-azide-resistant derivative of $E$. coli $\mathrm{J} 53\left(\mathrm{~J} 53_{\mathrm{RAZ}}\right)$. bla $\mathrm{NDM}$-containing transconjugants were obtained from all strains. However, in case of K. pneumoniae ABC137, the transconjugants obtained always carried multiple plasmid bands. Therefore, from one of these transconjugants, a crude plasmid extract was used to transform competent cells of $\mathrm{J} 53_{\mathrm{RAZ}}$ resulting in single, $b l a_{\mathrm{NDM}}$-carrying plasmid containing derivatives.

Attempts to conjugally transfer of bla $a_{\text {OXA-48-like }}$ carrying plasmid failed with all clinical isolates except for $\mathrm{ABC} 106$. However, we were successful to get single plasmid containing transformants carrying the respective $b l a_{\text {OXA-48-like }}$ genes in E. coli $\mathrm{DH} 5 \alpha$ using the crude plasmid extracts of $\mathrm{ABC} 120, \mathrm{ABC} 127, \mathrm{ABC} 128$, $\mathrm{ABC} 137$, and SA54.

Plasmid incompatibility types of carbapenemase-bearing plasmids were confirmed by PCR-based replicon typing, as well as by hybridization of the plasmid gels of the wild-type strains and single plasmid containing transconjugants or transformants. The size, incompatibility types, and genes co-transferring with the plasmids are shown in Table III. It should be noted that the approximately $110 \mathrm{~kb}$ NDM plasmid of SA54 harbored double replicase genes of incompatibility types FIb and FII. In this latter case, beyond PCR and hybridization, this incompatibility type was also confirmed by sequencing the respective region of the purified plasmid.

Transfer of the $b l a_{\text {OXA-48-like }}$ gene from OMABC109 was unsuccessful either by conjugation or by transformation. Therefore, S1 nuclease digestion of 


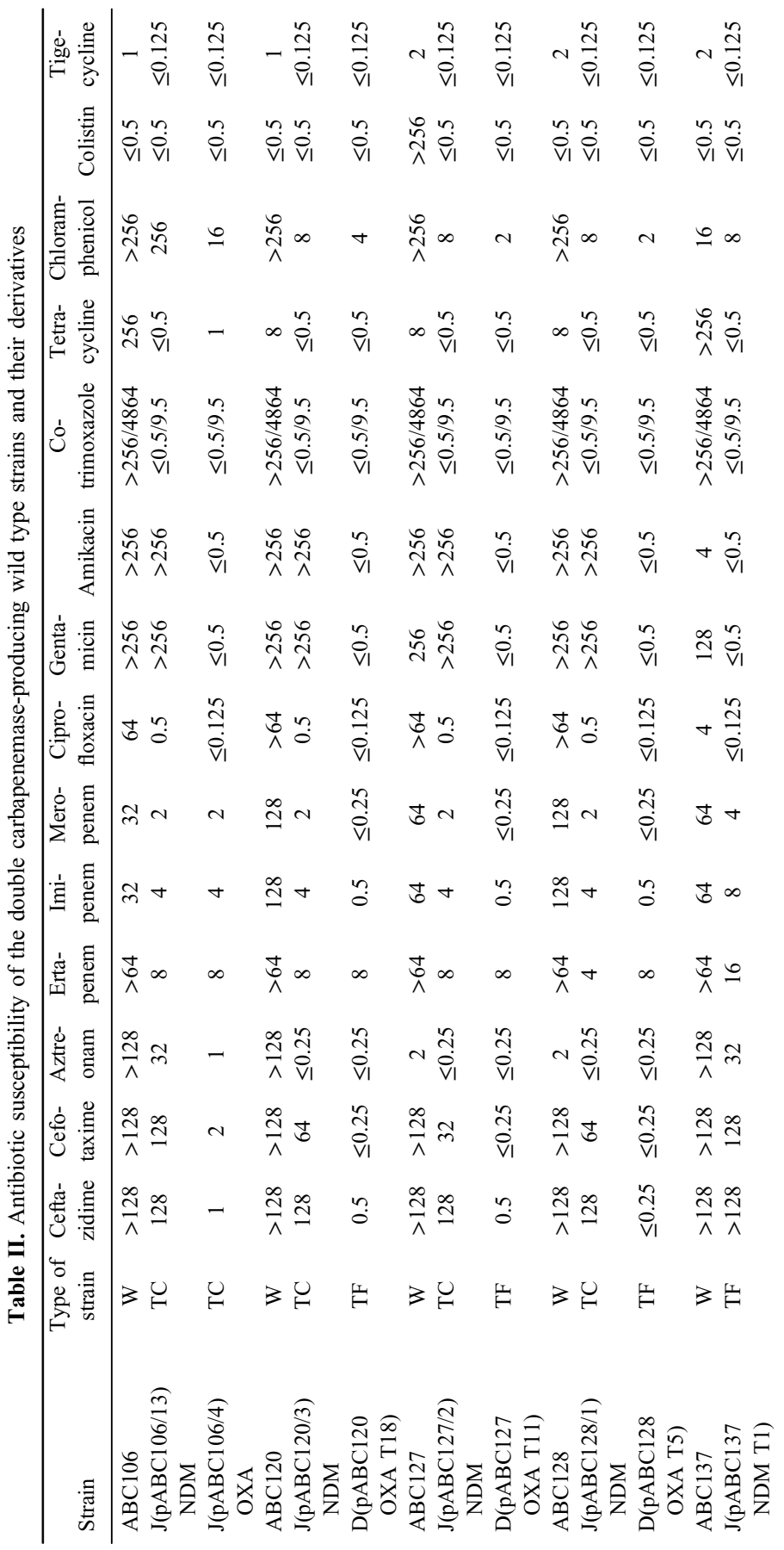




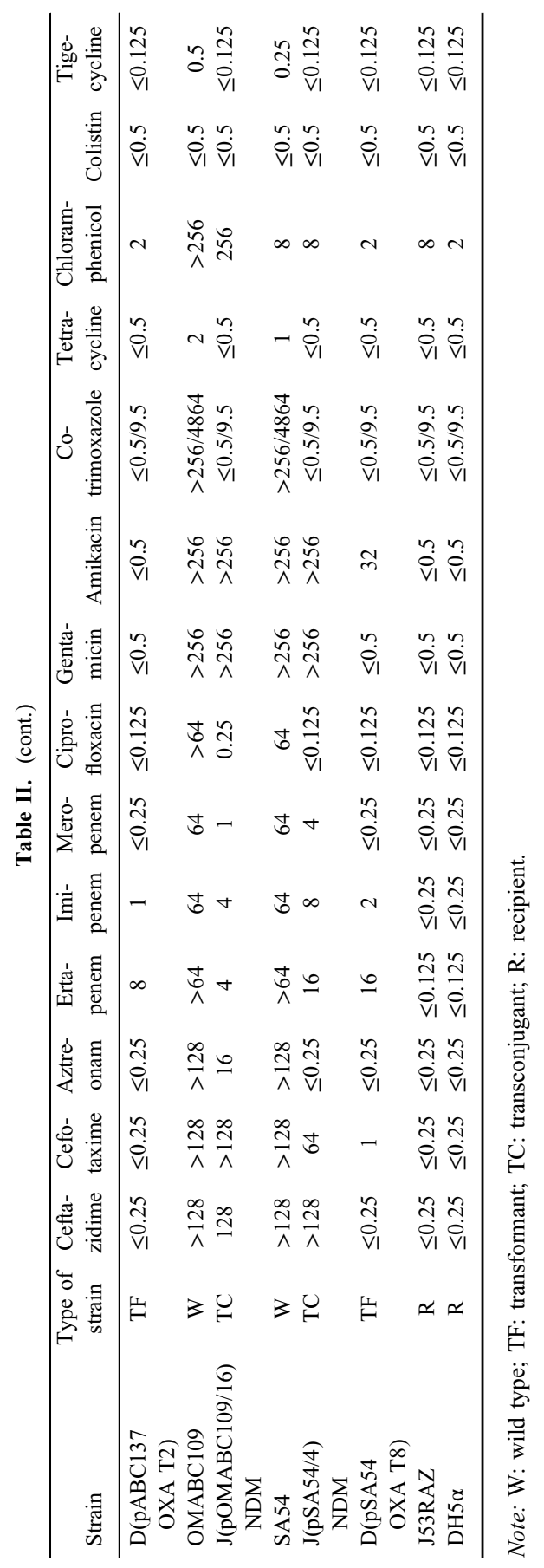




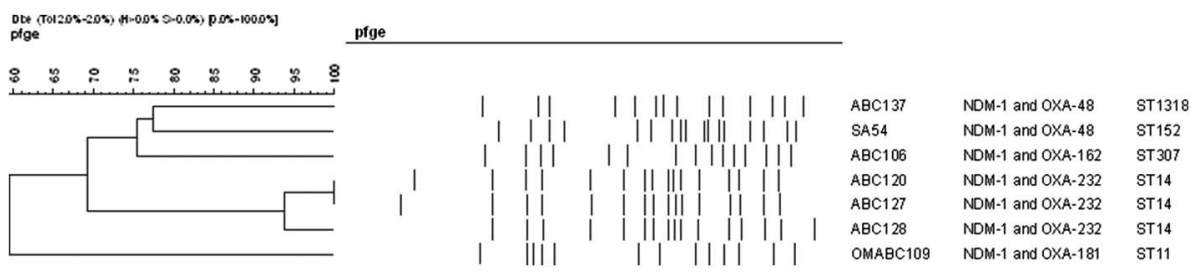

Figure 1. Comparison of pulsed-field gel electrophoresis (PFGE) patterns of double carbapenemaseproducing $K$. pneumoniae

OMABC109 was carried out and the gel was hybridized using an OXA-181 probe, which localized the gene on the chromosome.

The genetic surrounding of the bla $a_{N D M}$ and bla $a_{O X A-48-l i k e}$ genes

To compare the genetic support of the respective carbapenemase genes, the sequence of the regions flanking them was also determined.

The regions downstream of the $b l a_{\mathrm{NDM}-1}$ genes were identical in all isolates containing a bleomycin-resistance gene ble $e_{\mathrm{MBL}}$. Immediate upstream of the bla $a_{\mathrm{NDM}-1}$ gene, all plasmids contained varying sizes of the $3^{\prime}$ end of the ISAba125. Further upstream, the plasmids of the three clonally related isolates (ABC120, $\mathrm{ABC} 127$, and $\mathrm{ABC} 128$, respectively) were also identical containing an ISEc33 element. It was noteworthy that in the plasmids of ABC106 and OMABC109, while belonging to the same Inc type (HI1b) and exhibiting similar molecular mass $(>160 \mathrm{~kb})$, the region upstream of the carbapenemase gene contained a longer stretch of ISAba125 (254 bp instead of $101 \mathrm{bp}$ ) and an IS3000 element. The respective regions of the NDM plasmids of ABC137 and SA54 were completely different. In the former one, an IS5 element was identified, whereas the latter one contained a ribosomal methylase gene, $r m t C$ upstream of a 97-bp-long 3' end of ISAbal 25 transposase (Figure 2). The sequences surrounding of the $b_{\text {NDM-1 }}$ in ABC106, ABC120, ABC137, OMABC109, and SA54 isolates were deposited in the GenBank under accession numbers: MF774792, MF774793, MF774794, MF774795, and MF774796, respectively.

Although the class-D carbapenemases could not be mobilized by conjugation from six of the seven clinical isolates, mapping their genetic surrounding confirmed their location on mobile genetic elements. In pABC137-OXA, the bla $a_{\text {OXA-48 }}$ was located in a classical Tn1999 transposon, in pSA54-OXA, the same allele was located in a Tn1999 variant disrupted by IS $1 R$ both upstream and downstream of the carbapenemase gene. bla $a_{\text {OXA-162 }}$ was found in a $\operatorname{Tn} 1999.2$ variant in the conjugative pABC106-OXA. In the Omani isolate OMABC109, the 


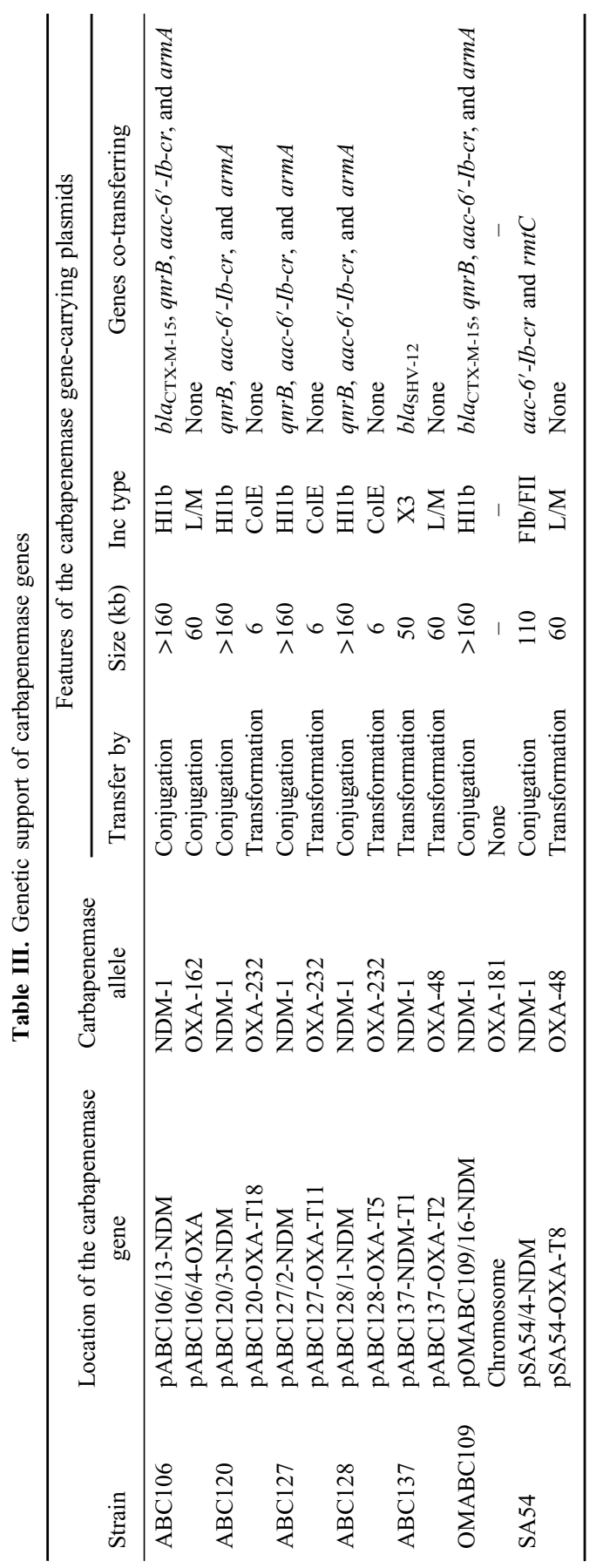




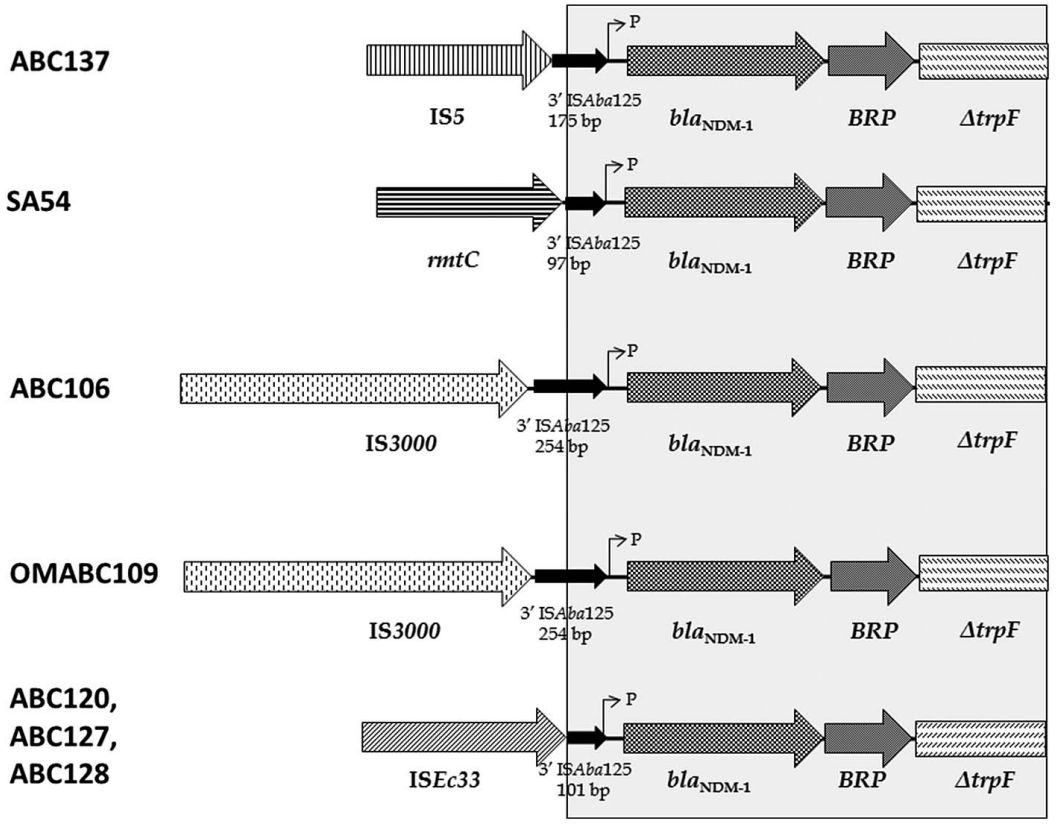

Figure 2. Genetic surrounding of $b l a_{\mathrm{NDM}-1}$ genes. The gray area indicates $100 \%$ identical regions

chromosomally located $b l a_{\text {OXA-181 }}$ was located in a Tn2013 with ISEcp 1 upstream of the carbapenemase gene (GenBank accession numbers: MF774788, MF774790, MF774787, and MF774789, respectively). In the clonally related isolates ABC120, $\mathrm{ABC} 127$, and $\mathrm{ABC} 128$, bla $_{\mathrm{OXA}-232}$ was located on 6,141-bp-long ColE-type nonconjugative plasmid (MF774791) as part of a truncated Tn2013 transposon in which only the 206-bp 3' end of the ISEcp I was present upstream of the bla $3_{\text {OXA-232 gene }}$ (Figure 3). The complete sequence of this plasmid was almost identical to the first sequenced pOXA-232 (JX423831).

\section{Plasmid RFLP}

As OMABC109 K. pneumoniae ST11 and ABC106 K. pneumoniae ST307, i.e., clonally unrelated strains, carried the $b l a_{\mathrm{NDM}-1}$ on IncHI1b-type plasmids of similar size, and the genetic surrounding of bla $a_{\mathrm{NDM}-1}$ was identical in both, we analyzed the similarity of these plasmids by RFLP. Although the restriction patterns of the two plasmids were not completely identical, they showed a considerable level of similarity (Figure 4). 


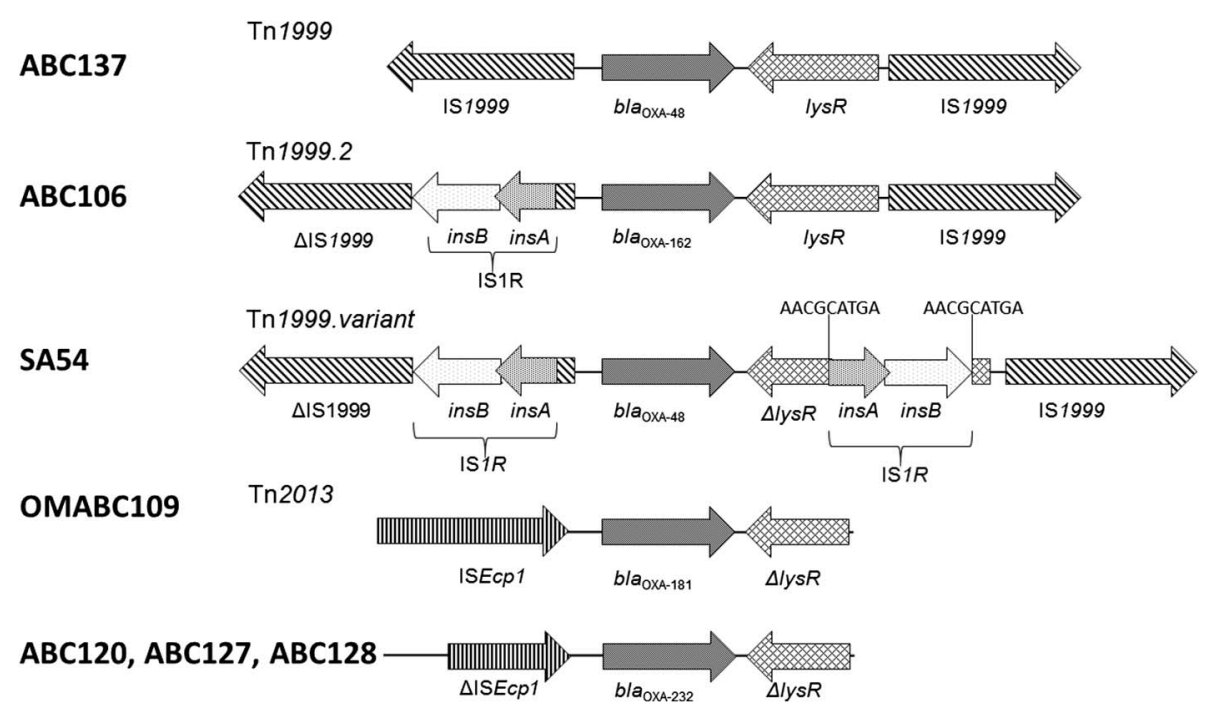

Figure 3. Genetic surrounding of bla $a_{\text {OXA-48-type }}$ genes

\section{Discussion}

The possible advantages provided by the seemingly redundant expression of multiple carbapenemases by a strain are a matter of speculation. One may assume that the varying substrate profiles and hydrolytic efficacies of the different carbapenemases (e.g., the considerably reduced activity of some class D enzymes against cephalosporins) may explain the better survival chances of cells carrying multiple carbapenemase genes. Alternatively, or even simultaneously, as the different carbapenemase genes often reside on different genomic entities (i.e., on different plasmids or some on the chromosome, while others on plasmids) may secure that, particularly in the absence of selective pressure, a single genetic event will not result in a carbapenem-susceptible phenotype.

Whatever the reason is, the rate of double carbapenemase producers, particularly NDM and OXA carbapenemase co-producing $K$. pneumoniae, is emerging: beyond sporadic isolates detected in the USA, Singapore, Denmark, and India $[5,6,8,9]$, clusters of clonally spreading such $K$. pneumoniae have been described in Saudi Arabia [20]. Furthermore, a cluster of NDM-5 and OXA-181 co-producing pan-drug-resistant $K$. pneumoniae ST147 was also reported from the UAE [21], showing a further evolutionary step in the development of antibiotic resistance in this species. The strains investigated in this study were isolated earlier than the above pan-drug-resistant isolates [21], and showed no genetic relatedness 


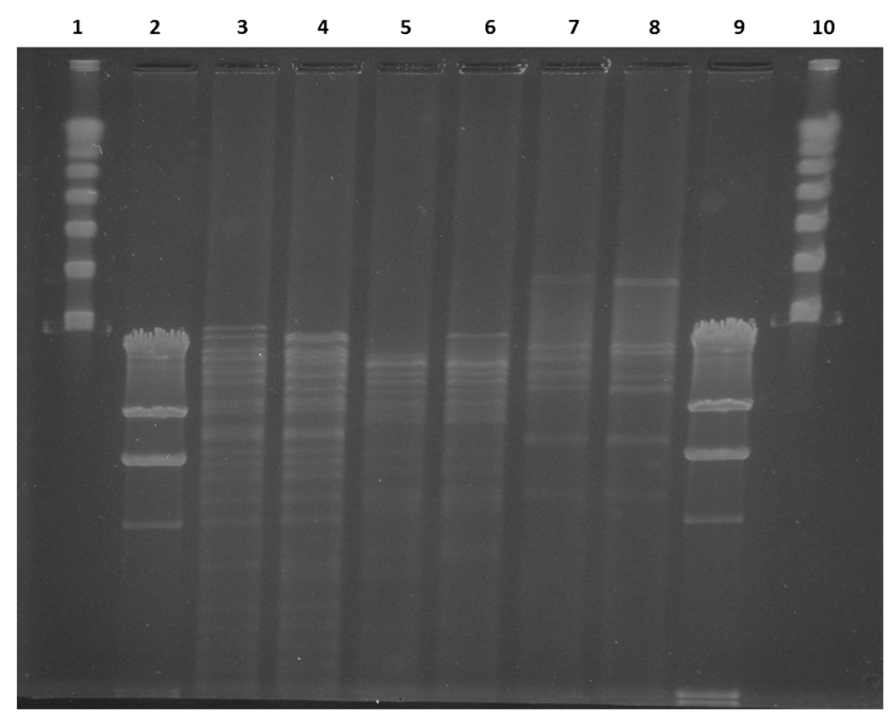

Figure 4. Plasmid restriction fragment length polymorphism of pABC106-NDM and pOMABC109NDM. Lane 1 and 10 Lambda concatemer (NEBiolabs), lanes 2 and 9 Lambda phage DNA digested with HindIII restriction endonuclease, lane 3 pABC106-NDM digested with BamHI, lane 4 pOMABC109-NDM digested with BamHI, lane 5 pABC106-NDM digested with SmaI, lane 6 pOMABC109-NDM digested with SmaI, lane 7 pABC106-NDM digested with $X b a \mathrm{I}$, and lane 8 pOMABC109-NDM digested with $\mathrm{XbaI}$

to those strains. According to the results presented here, the higher proportion of NDM- and OXA-48-like enzyme co-producing clinical isolates in the UAE observed by us earlier [10] could only be partially explained by clonal expansion of $K$. pneumoniae ST14, since two of the five strains from the UAE had no genetic relatedness to these isolates or to each other. However, it is interesting to note that double carbapenemase-producing isolates of the UAE harbored NDM plasmids similar to ones described earlier from the region. K. pneumoniae ABC137 carried $b l a_{\mathrm{NDM}-1}$ on an IncX3-type plasmid shown earlier to contribute to the spread of this carbapenemase in the UAE [12]. The other four isolates carried $b l a_{\mathrm{NDM}-1}$ on IncHI1b-type plasmids, which was encountered earlier in the UAE and also in Oman $[12,22]$. Furthermore, the highly similar pABC106-NDM of ABC106 K. pneumoniae ST307 from the UAE and pOMABC109-NDM of OMABC109 $K$. pneumoniae ST11 from Oman showed similarities to pNDM-MAR of a $K$. pneumoniae ST15 from Morocco, having identical incompatibility type and regions flanking $b l a_{\mathrm{NDM}-1}$, and also carrying $b l a_{\mathrm{CTX}-\mathrm{M}-15}$ and $q n r B 1$ genes [23]. The same incompatibility-type plasmid, but with different insertion element upstream of $b l a_{\mathrm{NDM}-1}$, was present in the three clonally related $K$. pneumoniae 
ST14. All these findings suggest the ability of this conjugative plasmid to establish itself in global multidrug-resistant clones of $K$. pneumoniae.

Contrary to the NDM-plasmids, the majority of OXA-plasmids encountered were not self-transmissible, although, with the exception of the blaxA-232 genes, all were located on mobile genetic elements known to be associated with the spread of these enzymes [24]. Nevertheless, bla $a_{\text {OXA-232 }}$ spread here due to the clonal expansion of $K$. pneumoniae ST14. This observation is in line with previous findings, as this particular carbapanemase was first described in K. pneumoniae ST14, and one of the first double carbapenemase-producing isolates reported was also a $K$. pneumoniae ST14 co-producing NDM-1 and OXA-232 [5, 25].

Characterization of these double carbapenemase-producing $K$. pneumoniae encountered in the early phase of spread of CRE in the UAE illustrates the complexity of the factors contributing to emergence of multidrug-resistant organisms. Unlike in a classical outbreak situation largely due to clonal spread, double carbapenemase-producing Enterobaceriaceae seem to have evolved due to horizontal transfer of mobile genetic elements encountered earlier either in the region or globally $[10,12,24]$. This latter way of transmission of antibiotic resistance threatens the efficiency of infection prevention, originally designed to control transfer of strains, i.e., their clonal spread and leaves antimicrobial stewardship exercised across the whole spectrum of human and veterinary medicine the only possible way to lessen the frequency of such horizontal gene transfer events.

\section{Acknowledgements}

This work was supported by grants UAEU UPAR-31M235 and CMHS$31 \mathrm{M} 251$ awarded to AS.

\section{Conflict of Interest}

No competing financial interests exist.

\section{References}

1. Iovleva, A., Doi, Y.: Carbapenem-resistant Enterobacteriaceae. Clin Lab Med 37, 303-315 (2017).

2. Logan, L. K., Weinstein, R. A. The epidemiology of carbapenem-resistant Enterobacteriaceae: The impact and evolution of a global menace. J Infect Dis 215, S28-S36 (2017). 
3. Pournaras, S., Poulou, A., Voulgari, E., Vrioni, G., Kristo, I., Tsakris, A.: Detection of the new metallo-beta-lactamase VIM-19 along with KPC-2, CMY-2 and CTX-M-15 in Klebsiella pneumoniae. J Antimicrob Chemother 65, 1604-1607 (2010).

4. Poulou, A., Voulgari, E., Vrioni, G., Xidopoulos, G., Pliagkos, A., Chatzipantazi, V., Markou, F., Tsakris, A.: Imported Klebsiella pneumoniae carbapenemase-producing $K$. pneumoniae clones in a Greek hospital: Impact of infection control measures for restraining their dissemination. J Clin Microbiol 50, 2618-2623 (2012).

5. Doi, Y., Hazen, T. H., Boitano, M., Tsai, Y. C., Clark, T. A., Korlach, J., Rasko, D. A.: Whole-genome assembly of Klebsiella pneumoniae coproducing NDM-1 and OXA-232 carbapenemases using single-molecule, real-time sequencing. Antimicrob Agents Chemother 58, 5947-5953 (2014).

6. Balm, M. N., La, M. V., Krishnan, P., Jureen, R., Lin, R. T., Teo, J. W.: Emergence of Klebsiella pneumoniae co-producing NDM-type and OXA-181 carbapenemases. Clin Microbiol Infect 19, E421-E423 (2013).

7. Karabay, O., Altindis, M., Koroglu, M., Karatuna, O., Aydemir, O. A., Erdem, A. F.: The carbapenem-resistant Enterobacteriaceae threat is growing: NDM-1 epidemic at a training hospital in Turkey. Ann Clin Microbiol Antimicrob 15, 6 (2016).

8. Hammerum, A. M., Littauer, P., Hansen, F.: Detection of Klebsiella pneumoniae co-producing NDM-7 and OXA-181, Escherichia coli producing NDM-5 and Acinetobacter baumannii producing OXA-23 in a single patient. Int J Antimicrob Agents 46, 597-598 (2015).

9. Veeraraghavan, B., Shankar, C., Karunasree, S., Kumari, S., Ravi, R., Ralph, R.: Carbapenem resistant Klebsiella pneumoniae isolated from bloodstream infection: Indian experience. Pathogens and Global Health 111, 240-246 (2017).

10. Sonnevend, A., Ghazawi, A. A., Hashmey, R., Jamal, W., Rotimi, V. O., Shibl, A. M., Al-Jardani, A., Al-Abri, S. S., Tariq, W. U., Weber, S., Pál, T.: Characterization of carbapenem-resistant Enterobacteriaceae with high rate of autochthonous transmission in the Arabian Peninsula. PLoS One 10, e0131372 (2015).

11. CLSI: Performance Standard for Antimicrobial Susceptibility testing (M100-S24). Clinical and Laboratory Standard Institute, Wayne, PA, 2014.

12. Sonnevend, A., Al Baloushi, A., Ghazawi, A., Hashmey, R., Girgis, S., Hamadeh, M. B., Al Haj, M., Pal, T.: Emergence and spread of NDM-1 producer Enterobacteriaceae with contribution of IncX3 plasmids in the United Arab Emirates. J Med Microbiol 62, 1044-1050 (2013).

13. Sonnevend, A., Ghazawi, A., Yahfoufi, N., Al-Baloushi, A., Hashmey, R., Mathew, M., Tariq, W. Z., Pal, T.: VIM-4 carbapenemase-producing Enterobacter cloacae in the United Arab Emirates. Clin Microbiol Infect 18, E494-E496 (2012).

14. Kado, C. I., Liu, S. T.: Rapid procedure for detection and isolation of large and small plasmids. J Bacteriol 145, 1365-1373 (1981).

15. Macrina, F. L., Kopecko, D. J., Jones, K. R., Ayers, D. J., McCowen, S. M.: A multiple plasmid-containing Escherichia coli strain: Convenient source of size reference plasmid molecules. Plasmid 1, 417-420 (1978).

16. Threlfall, E. J., Rowe, B., Ferguson, J. L., Ward, L. R.: Characterization of plasmids conferring resistance to gentamicin and apramycin in strains of Salmonella typhimurium phage type 204c isolated in Britain. J Hyg 97, 419-426 (1986).

17. Carattoli, A., Bertini, A., Villa, L., Falbo, V., Hopkins, K. L., Threlfall, E. J.: Identification of plasmids by PCR-based replicon typing. J Microbiol Methods 63, 219-228 (2005). 
18. Johnson, T. J., Bielak, E. M., Fortini, D., Hansen, L. H., Hasman, H., Debroy, C., Nolan, L. K., Carattoli, A.: Expansion of the IncX plasmid family for improved identification and typing of novel plasmids in drug-resistant Enterobacteriaceae. Plasmid 68, 43-50 (2012).

19. Ghazawi, A., Sonnevend, A., Bonnin, R. A., Poirel, L., Nordmann, P., Hashmey, R., Rizvi, T. A., Hamadeh, M. B., Pal, T.: NDM-2 carbapenemase-producing Acinetobacter baumannii in the United Arab Emirates. Clin Microbiol Infect 18, E34-E36 (2012).

20. Zowawi, H. M., Sartor, A. L., Balkhy, H. H., Walsh, T. R., Al Johani, S. M., AlJindan, R. Y., Alfaresi, M., Ibrahim, E., Sl-Jardani, A., AL-Abri, S., Al Salman, J., Dashti, A. A., Kutbi, A. H., SCHlebusch, S., Sidjabat, E., Paterson, D. L.: Molecular characterization of carbapenemase-producing Escherichia coli and Klebsiella pneumoniae in the countries of the Gulf cooperation council: Dominance of OXA-48 and NDM producers. Antimicrob Agents Chemother 58, 3085-3090 (2014).

21. Sonnevend, A., Ghazawi, A., Hashmey, R., Haidermota, A., Girgis, S., Alfaresi, M., Omar, M., Paterson, D. L., Zowawi, H. M., Pal, T.: Multihospital occurrence of pan-resistant Klebsiella pneumoniae sequence type 147 with an ISEcp1-directed blaOXA-181 insertion in the mgrB gene in the United Arab Emirates. Antimicrob Agents Chemother 61, e00418-17 (2017).

22. Dortet, L., Poirel, L., Al Yaqoubi, F., Nordmann, P.: NDM-1, OXA-48 and OXA-181 carbapenemase-producing Enterobacteriaceae in Sultanate of Oman. Clin Microbiol Infect 18, E144-E148 (2012).

23. Villa, L., Poirel, L., Nordmann, P., Carta, C., Carattoli, A.: Complete sequencing of an IncH plasmid carrying the blaNDM-1, blaCTX-M-15 and qnrB1 genes. J Antimicrob Chemother 67, 1645-1650 (2012).

24. Poirel, L., Potron, A., Nordmann, P.: OXA-48-like carbapenemases: The phantom menace. J Antimicrob Chemother 67, 1597-1606 (2012).

25. Potron, A., Rondinaud, E., Poirel, L., Belmonte, O., Boyer, S., Camiade, S., Nordmann, P.: Genetic and biochemical characterisation of OXA-232, a carbapenem-hydrolysing class D beta-lactamase from Enterobacteriaceae. Int J Antimicrob Agents 41, 325-329 (2013). 